\title{
Surface passivation by graphene in the lubrication of iron: A comparison with bronze
}

\author{
Diego Marchetto $^{\mathrm{a}, \mathrm{b}, \mathrm{c}, *}$, Paolo Restuccia ${ }^{\mathrm{a}}$, Antonio Ballestrazzi ${ }^{\mathrm{a}, \mathrm{c}}$, M. C. Righi ${ }^{\mathrm{a}, \mathrm{b}}$, Alberto \\ Rota $^{\mathrm{c}}$, Sergio Valeri ${ }^{\mathrm{a}, \mathrm{b}, \mathrm{c}}$ \\ ${ }^{a}$ Dipartimento di Scienze Fisiche, Informatiche e Matematiche, Università di Modena e Reggio Emilia, \\ Via Campi 213/A, 41125 Modena, Italy \\ ${ }^{b}$ CNR-Institute of Nanoscience, S3 Center, Via Campi 213/A, 41125 Modena, Italy \\ ${ }^{c}$ Centro Interdipartimentale per la Ricerca Applicata e i Servizi nel Settore della Meccanica Avanzata e \\ della Motoristica, Universita di Modena e Reggio Emilia, Via Vivarelli 2, 41125 Modena, Italy
}

\begin{abstract}
It has been recently reported that graphene is able to significantly reduce the friction coefficient of steel-on-steel sliding contacts. The microscopic origin of this behavior has been attributed to the mechanical action of load carrying capacity. However, a recent work highlighted the importance of the chemical action of graphene. According to this work graphene reduces the adhesion of iron interfaces by reducing the surface energy thanks to a passivation effect. The aim of the present work is to clarify the still debated lubricating behavior of graphene flakes. We perform pin-on-disc experiments using liquid dispersed graphene solution as a lubricant. Two different materials, pure iron and bronze are tested against 100Cr6 steel. Raman spectroscopy is used to analyze the surfaces after the friction tests. The results of these tests prove that graphene flakes have a beneficial effect on the friction coefficient. At the same time they show a tendency of graphene to passivate the native iron surfaces that are exposed during sliding as a consequence of wear.
\end{abstract}

\section{Introduction}

The recent need for friction and wear control in mechanical high-technology applications has taken to the introduction of layered materials $\left(\mathrm{MoS}_{2}, \mathrm{HBN}\right.$, graphite) in coatings, addi-

\footnotetext{
*Corresponding author

Email address: diego.marchetto@unimore.it (Diego Marchetto)
} 
tives, liquid and solid lubricants [1-7]. As a further scientific and technological improvement the potential of graphene as a lubricant is currently under investigation. The reduction of friction obtained with the application of a layer of graphene is studied at different scales with different methods both theoretically [8-21] and experimentally [22-45].

While the majority of the research at the nanoscale investigates the properties of graphene as a solid atomic-thick coating [22-29] at the macroscale graphene is studied also as colloidal liquid lubricant [30-37]. In few recent works, Berman et al. successfully used graphene to lubricate a steel-steel sliding contact [31, 32, 42, 43]. Graphene flakes dispersed in an alcohol solution were applied to a steel surface and tested by tribometer. In humid environment the reduction of friction was dramatic $[42,43]$. While the steel on steel coefficient of friction is usually around 0.9 in the presence of graphene it dropped to about 0.2 [42]. In inert environment the same values for the friction coefficient were found together with a marked reduction of wear $[42,43]$. The lifetime of the graphene layer and therefore of the lubricated regime decreased with the interface pressure [43]. The short lifetime of graphene layers at high loads has been observed in other works [20, 29].

A theoretical work on the processes governing the tribology of metal-supported graphene was published in 2014 by Klemenz et al. [20]. They combined atomistic simulation of nanoindentation and AFM experiments on graphene covered Pt(111) surfaces. The reduction of friction coefficient achieved with the application of graphene was explained by the authors with the ability of graphene to increase the load carrying capacity of the surface [20]. However, when graphene gets damaged it loses its lubricating properties [20].

Restuccia et al. instead propose that the lubricating behavior of graphene is due to its ability to passivate the steel surfaces [46]. They show that the carbon dangling bonds of graphene chemically adsorbs on native iron surfaces produced during sliding screening the metal-metal interaction. Due to this behavior the atomic-thick carbon layer is able to reduce the interfacial adhesion and shear strength [46].

Therefore while the work of Klemenz et al. takes into account mechanical lubrication [20], the one of Restuccia et al. involves tribochemical lubrication [46].

With this paper we add a new piece of information for understanding the lubricating

(C) 2017. This manuscript version is made available under the CC-BY-NC-ND 4.0 license http://creativecommons.org/licenses/by-nc-nd/4.0/ 
properties of graphene by analyzing the role played by the surface chemistry from an experimental point of view. We perform ball-on-disc friction tests on pure iron and on bronze using a solution of graphene flakes in ethanol. Performing Raman analysis on the samples (discs and balls) before and after the tests we analyze the status of the graphene flakes and identify their main function. It must be said that the case described by Klemenz et al. is different from the one analyzed here. They studied epitaxially grown graphene without any defects while we study the behavior of deposited graphene flakes.

\section{Materials and methods}

The tribological tests are performed by means of a commercial tribometer CETR UMT3 in ball-on-disc configuration similarly to previous studies on liquid dispersed solution of graphene [31, 32, 37, 42, 43]. The ball used for all tests is $4 \mathrm{~mm}$ in diameter made out of steel 100Cr6.

The tested samples are made of iron (99.98\% pure) and bronze ( $98 \% \mathrm{Cu}$ and $2 \% \mathrm{Sn})$. The discs are all polished in order to obtain a $30 \mathrm{~nm}$ surface average roughness $R_{a}$.

Tests are performed maintaining a tangential sliding velocity of $100 \mathrm{~mm} / \mathrm{s}$ (at a radius of $10 \mathrm{~mm}$ ) and the applied load is $1 \mathrm{~N}$. This load produces a nominal average pressure of about $0.4 \mathrm{GPa}$ (Hertzian pressure). All tests are made at room temperature with a $R H$ humidity of about $50 \%$.

For the tests with graphene we used a commercial liquid dispersed solution of graphene single layers (Graphene Supermarket). The dispersion is in ethanol with a concentration of the graphene flakes of $1 \mathrm{mg} / \mathrm{L}$. The flakes should not present either oxidation or surfactants. The average flake size is $550 \mathrm{~nm}$ (150-3000) nm. Tests performed by AFM on some flakes deposited on $\mathrm{SiO}_{2}$ show that the flakes are actually multilayered and present defects and contamination (see Fig. 1a and b). In Fig. 1c the Raman spectrum of graphene flakes deposited on a clean iron surface is shown. The D, G and 2D peaks of graphene are present. The intensity of the $\mathrm{D}$ peak (related to the breathing mode of the sp2 atoms) indicates that the amount of crystalline defects, edges or oxidation is rather strong for such commercial flakes, and probably fluctuating among different batches (see the qualitatively

(C) 2017. This manuscript version is made available under the CC-BY-NC-ND 4.0 license http://creativecommons.org/licenses/by-nc-nd/4.0/ 
different spectrum in Fig. 1b of [42], for the very same graphene solution). The spectrum also confirms that the flakes are not single layer but more probably made of two or three layers. This is compatible with previous studies [37, 47].
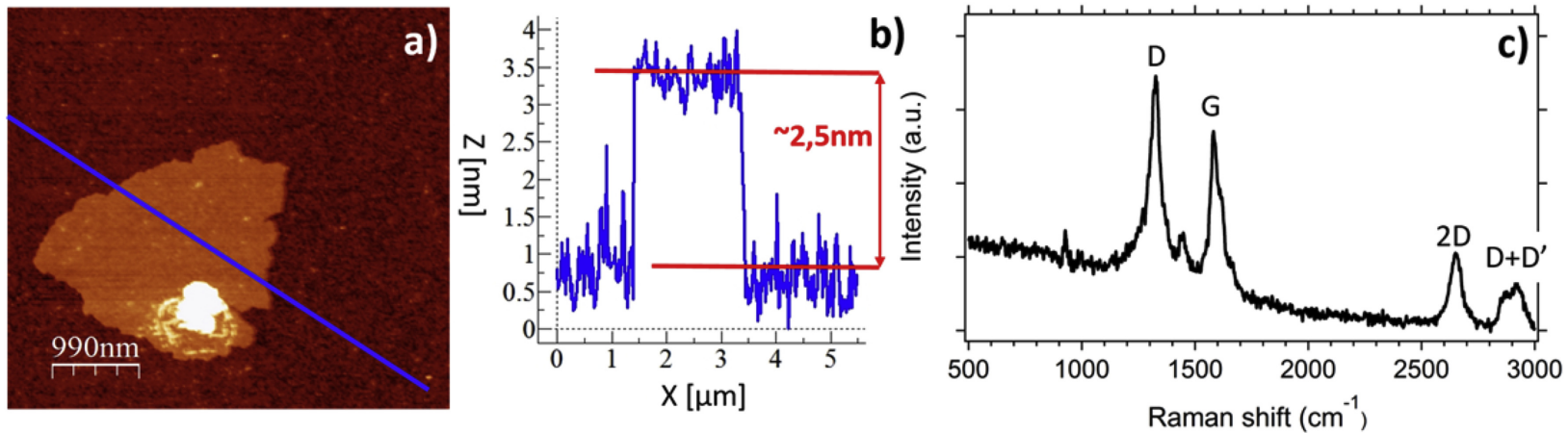

Figure 1: a) AFM picture of a flake of graphene deposited on $\mathrm{SiO}_{2}$. The image shows that some kind of contamination is present. The lateral dimension correspond to the specifics. b) Profile. The thickness is quite high and corresponds to about three layer of graphene. c) Raman spectrum of graphene deposited on a clean iron surface. The characteristic peaks D, G and 2D (at 1330, 1590 and $2650 \mathrm{~cm}^{-1}$ ) of graphene are observed. The intensity of the $\mathrm{D}$ peak proves that the flakes present a large amount of defects, edges or partial oxidation.

Drops of graphene solution are applied directly on the wear track during the friction tests. Each drop is about $0.05 \mathrm{ml}$. Before the tests some drops are applied and let exsiccate on the surface both of Fe samples and of bronze ones. This procedure already tested by Berman et al. produces a layer of graphene flakes on the sample surfaces $[42,43]$.

The surfaces are analyzed by Raman spectroscopy [He-Ne Laser, $632.81 \mathrm{~nm}$ e Jobin Yvon] inside and outside the wear-tracks before and after the tests.

\section{Experimental results}

An example of friction results is reported in Fig. 2a. The graph shows a comparison between tests performed in "dry" conditions, a test performed adding drops of graphene solution and a test performed adding drops of pure ethanol. Drops are added in both cases every two minutes in the amount of three at the first step, three at second, two at the third

(C) 2017. This manuscript version is made available under the CC-BY-NC-ND 4.0 license http://creativecommons.org/licenses/by-nc-nd/4.0/ 
and one at the fourth step. In dry conditions the friction coefficient after the running-in becomes stable around a value of about 0.46 . The test performed adding graphene show a completely different behavior. The COF starts at a value of 0.05 and during the running-in phase rises to 0.32 . This is due to the presence of the graphene layer produced before the test. After three minutes the first three drops are applied and the COF goes down to 0.15. Although with some oscillations the COF does not move from this value for the rest of the test and drop steps.
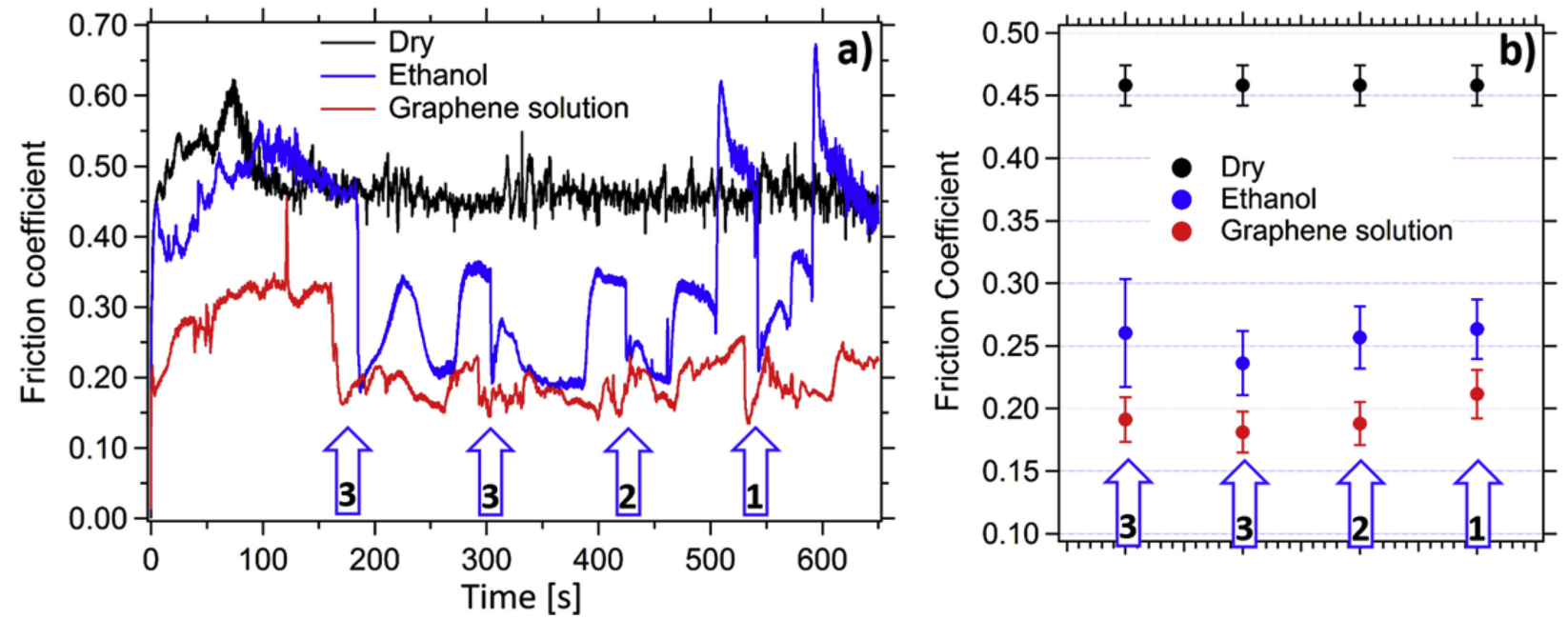

Figure 2: Friction coefficient results. a) example of the friction coefficient behavior in a dry contact (black), in a contact lubricated by ethanol (blue) and in a contact lubricated by ethanol solution containing graphene (red). The arrows represent the application time when droplets are poured, the number of which is indicated by the arrow; b) Average values of the friction coefficient obtained averaging the data of the tests after the solution application.

Using ethanol drops (blue line Fig. 2a) the behavior is different. The friction reduction after applying few drops of ethanol is evident, but the coefficient rises as soon as the ethanol evaporates. When just one drop is poured (last step) the evaporation time is quite short and the lubrication process lasts for few cycles only.

In Fig. $2 \mathrm{~b}$ the average values of the friction coefficient are reported. They are calculated by averaging the data recorded between the pouring moments. For ethanol the data obtained after the evaporation of the liquid were excluded from the average. In this way it is possible

(C) 2017. This manuscript version is made available under the CC-BY-NC-ND 4.0 license http://creativecommons.org/licenses/by-nc-nd/4.0/ 
to compare the lubrication effect of liquid ethanol with that of ethanol containing graphene flakes. As can be seen in Fig. 2a, the friction coefficient in dry conditions is about 0.46. Introducing ethanol we obtain a $\mu=0.24$ with some fluctuations. Graphene flakes lower the friction coefficient to a value of $\mu=0.19$. In few occasions the pure ethanol and the graphene-containing solution have comparable values (mainly due to big fluctuations in the ethanol $\mu$ values) but considering the whole picture it is clear that graphene not only improves the duration of the low friction regime, but also improves the lubricating effect in terms of absolute value as well.

The Raman analysis performed on the samples is reported in Fig. 3. Since the friction test is carried out after the solution is completely evaporated from the sample and the friction coefficient is still low (at a value of about 0.16) it is possible to investigate the presence of graphene both on the wear-tracks and on the ball. The results reported in Fig. 3a show that two different kinds of spectra can be found within the wear track. One spectrum corresponds to iron oxide. No graphene is visible. This can be associated with the oxidation of the surface and the formation of wear debris due to sliding. The second spectrum clearly shows the presence of graphene. The $2 \mathrm{D}$ peak at $2646 \mathrm{~cm}^{-1}$ is clearly visible and so are D and $\mathrm{G}$ peaks proving that the layer is not single and that defects are definitely present. The $2 \mathrm{D}$ peak is smaller than the one measured on a pristine layer (Fig. 1) while D and G are much more intense. The D peak in particular reveals that the flakes of graphene are highly damaged. This should not be a surprise considering that those flakes were laying in between the two sliding surfaces standing a pressure of several hundred MPa. A similar situation is shown by Mao et al. for flakes applied in electric contacts [39].

In Fig. 3b the Raman spectrum from the ball shows that on the sphere in the contact area there is actually no graphene. D and G peaks are barely visible and the $2 \mathrm{D}$ peak is not visible at all, as it happens in amorphous carbon [39]. So on the ball there is just a transfer of carbon residues from the grinding of graphene during sliding.

The ball-on-disc tests are repeated on a bronze disc. The same set-up described above is used. The results are reported in Fig. 4a. The ethanol on bronze gives almost none lubrication. We observe a drop in the friction coefficient but the intensity and duration of

(C) 2017. This manuscript version is made available under the CC-BY-NC-ND 4.0 license http://creativecommons.org/licenses/by-nc-nd/4.0/ 

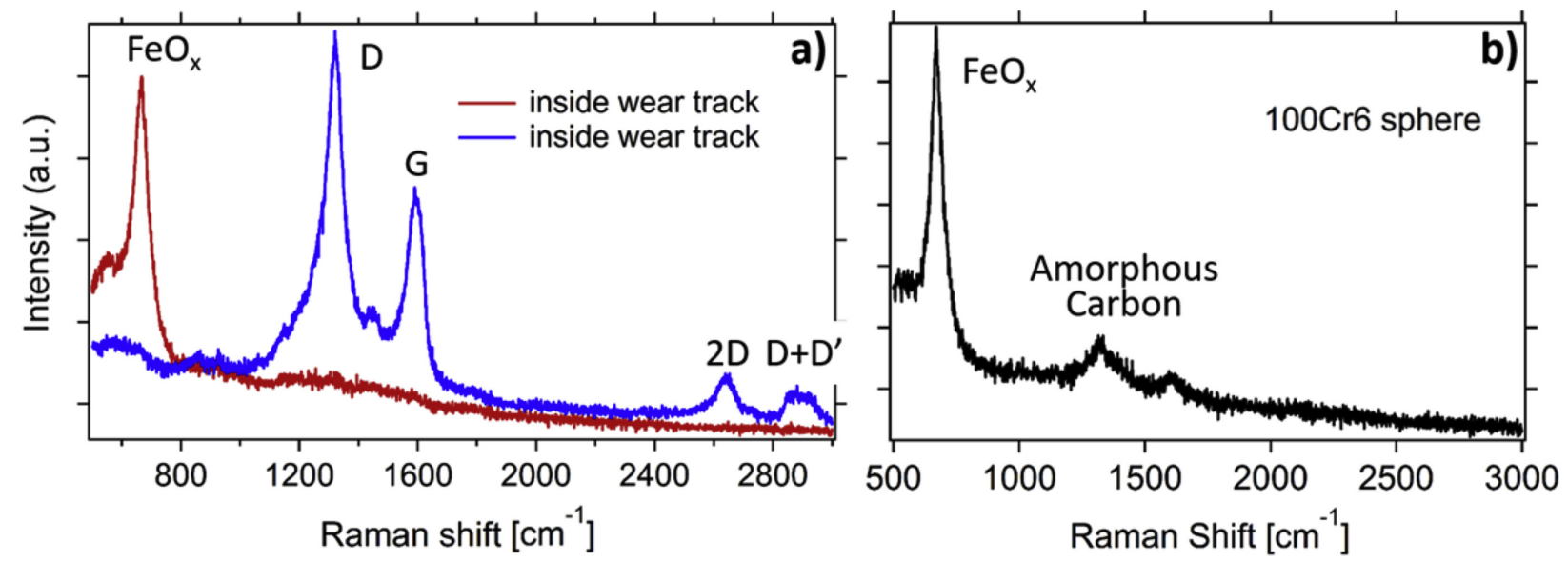

Figure 3: a) Raman spectroscopy of a wear track on Fe samples. The friction tests was stopped few minutes after pouring graphene drops. Ethanol was completely evaporated and the friction coefficient was still low around $\mu=0.16$. The red curve show no carbon at all, while the blue curve shows a clear presence of graphene. b) Raman spectroscopy of the sphere used for the test. The shape of the curve shows the presence of iron oxide and amorphous carbon.

it is very much different from those observed on iron. After a few cycles of lubrication the friction coefficient rises back to the "dry" values. The system lubricated by the graphene solution shows a stable value of $\mu=0.15$ from the very beginning. Only after putting the first three drops some instabilities in the friction appear.

The Raman spectra obtained on the bronze sample are reported in Fig. 4b. The weartrack corresponds to a test stopped in the low friction regime after drops of the graphenecontaining solution have been poured and the ethanol is evaporated. The curves show that graphene is present outside the track (red curve) but not inside (blue curve). Measurements performed on the ball inside the contact area show very intense peaks of graphene (black curve). The graphene D peak is quite high (as well as on iron sample) indicating that the graphene flakes attached to the ball contain defects produced by the friction process.

\section{Discussion}

The results reported above give us a quite clear picture of the behavior of the graphene flakes. Starting from Fig. 3, the presence of graphene in the wear track verified by the (C) 2017. This manuscript version is made available under the CC-BY-NC-ND 4.0 license http://creativecommons.org/licenses/by-nc-nd/4.0/ 

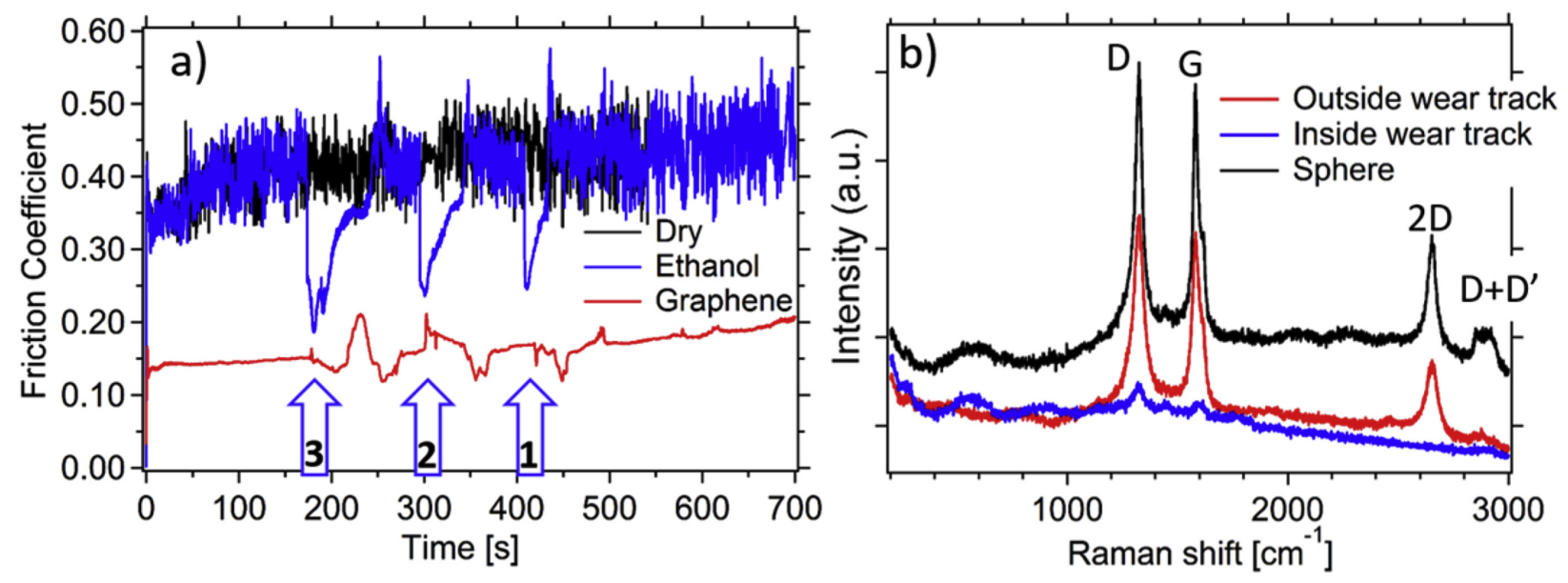

Figure 4: a) Pin-on-disc results obtained on a bronze disc. The arrows represent the number of drops (indicated on the arrow) and the instant at which they are poured. The black line represents the test in dry conditions which is shorter; b) Raman spectra obtained on the bronze sample and on the ball.

Raman spectroscopy confirms that the flakes have a lubricating effect. The Raman analysis performed on wear tracks after the friction coefficient is back to "dry values" $(\mu=0.45)$ do not show any presence of graphene. These data are close to the results presented in literature by Berman et al. [31, 32, 42, 43]. On the other hand the duration of the lubricating effect is totally different. Berman et al. report thousands of cycles at low friction coefficient while in our experiment we reach a few hundred at best (once we stop pouring solution drops). This difference is ascribable to the quality of graphene. The flakes used for this experiment are highly defected and partially oxidized while the graphene used by Berman et al. is of a much higher quality. This statement is confirmed by the comparison of the Raman spectrum reported in Fig. 1c and the one reported by Berman [43].

The presence of completely uncovered areas in the wear track on which the Raman spectrum shows only the iron oxide peak, reveals that the coverage is not complete. Furthermore the Raman spectrum of the graphene flakes presents a D peak that is double the size of the $\mathrm{G}$ one. This is due to a presence of defects. Finally the Raman spectrum of the sphere does not show any presence of graphene. This last result is a bit surprising. We would indeed expect graphene to cover both surfaces.

(C) 2017. This manuscript version is made available under the CC-BY-NC-ND 4.0 license http://creativecommons.org/licenses/by-nc-nd/4.0/ 
The picture becomes much clearer when the results of Fig. 3 are compared to the results of Fig. 4. On bronze the behavior of graphene is completely different. No carbon material is found in the wear track although the friction coefficient induced by graphene lubrication is even more stable than the one on Fe. Graphene on the sample is found only outside the wear track as expected (from the layer are produced before the tests). Instead a very clear signature of graphene is visible on the 100Cr6 sphere inside the area of contact. The intensity of the peaks D, G and 2D is comparable to that of the peaks produced by the initial layer on the bronze sample (Fig. 4). This fact implies that the graphene on the ball is not as damaged as the graphene found in the wear-track on the Fe sample.

The fact that graphene is found on the sphere and not on bronze indicates that graphene flakes adsorb most preferably on iron than on bronze. This experimental observation can be explained by comparing the graphene adsorption energy on iron and copper (the bronze sample used in the experiment contains $98 \%$ copper). The binding energy per carbon atom obtained by DFT calculations within the local density approximation (LDA) corresponds to $36 \mathrm{meV}$ on copper and to 134 on iron (full calculations are shown in Supplementary materials). The comparison of the binding energies and the equilibrium distances, $3.2 \AA$ on copper and $2.04 \AA$ on iron, indicates that there is a marked difference in the nature of the interactions: graphene physisorbs on copper, while it chemisorbs on iron, where the pi orbital of graphene hybridize with the $\mathrm{d}$ states of iron that are not fully occupied. The different nature of the interactions, is also reflected in the electronic structure of adsorbed graphene, which preserve its main feature on copper, while it is strongly perturbed on iron [48, 49].

Graphene does not cover the entire surface but work as an oil additive. During sliding of the steel sphere against the discs (both bronze and $\mathrm{Fe}$ ) some wear occurs. In the case of pure iron the majority of the wear is located on the surface of the disc. Fe is softer than steel and the superficial iron oxide layer is continuously worn out.

This process uncovers native Fe surfaces, which are chemically very active. As explained by Restuccia et al. [46] the graphene flakes dispersed in the solution stick to this spots reducing the surface energy and therefore the friction coefficient. This behavior explains the reduction of friction and the instability of the friction coefficient. Indeed, the coating is not

(C) 2017. This manuscript version is made available under the CC-BY-NC-ND 4.0 license http://creativecommons.org/licenses/by-nc-nd/4.0/ 
complete and once the ethanol is completely evaporated the flakes cannot move anymore. Therefore the sphere asperities encounter areas with low friction due to the presence of graphene covering Fe and areas at higher friction with uncovered Fe bonds or iron oxide. The fact that the Raman spectra obtained from these tests show very damaged graphene could be due both by the presence of an oxide layer on iron that induces oxidation also in graphene during sliding and by the defects originating from the high pressure at the asperity-asperity contacts.

On the bronze sample the flakes do not stick to the wear track. Raman spectrum only shows the presence of some amorphous carbon due to the presence of carbon material (coming from graphene, the environment, and ethanol). On the other hand the 100Cr6 steel sphere is well covered by graphene. The flakes bind to the Fe bonds on the ball surface (100Cr6 is about $97 \%$ iron). The better stability and duration of the low friction regime obtained on bronze compared to steel is probably due to the higher "graphene flakes concentration/Fe active bonds" ratio. The graphene coverage is higher and therefore the surface energy is lower. This result is in good agreement with the outcome of the analysis on the effects of graphene coverage performed in Ref. 46 by means of first principles calculations. As a further proof of this behavior, an analysis of some of the wear tracks (reported in Supplementary materials) shows that the wear reduction induced by the graphene lubrication in bronze tests is higher than that observed in tests performed on iron.

\section{Conclusions}

We performed tribological experiments and spectroscopic analysis to shed light into the chemical mechanisms underlying lubricating effect of graphene. An ethanol solution containing graphene flakes was used to lubricate a steel-iron and a steel-bronze interface in ball-on-disc friction tests. The reduction of friction coefficient due to graphene was compared to the one obtained with the use of pure ethanol and to the "dry" condition. A reduction of friction ascribable to graphene was measured both in terms of absolute value and in terms of duration compared to the pure ethanol.

(C) 2017. This manuscript version is made available under the CC-BY-NC-ND 4.0 license http://creativecommons.org/licenses/by-nc-nd/4.0/ 
The Raman spectroscopy performed on the disc surfaces and on the contact area of the balls showed that graphene flakes binds to iron rather than other materials. Indeed in the steel-iron sliding contact graphene was found on the Fe surface while in the steel-bronze system graphene bound to the active region of native iron which were produced on the steel ball by rubbing. We were therefore able to show that the lubrication due to the graphene flakes is related to the chemical passivation of iron by graphene.

The difference between the friction coefficients obtained on pure iron and on bronze were attributed to the different coverage of the surfaces. In the case of steel ball sliding on bronze the small contact area of the sphere is quickly covered by graphene flakes inducing a more stable and durable lubricating effect.

\section{Acknowledgments}

The authors gratefully acknowledge the support by Cost Action MP1303 "Understanding and controlling nano and mesoscale friction' and FCRMo for support through the project SIME 1013.0650 (call "Applied Research for Innovation"). We thank Renato Buzio from CNR-SPIN for useful discussion.

\section{Appendix A. Supplementary data}

Supplementary data related to this article can be found at link.

\section{References}

\section{References}

[1] T. W. Scharf, S. V. Prasad, Solid lubricants: a review, Journal of Materials Science 48 (2) (2013) 511-531. doi:10.1007/s10853-012-7038-2.

URL https://link. springer.com/article/10.1007\%2Fs10853-012-7038-2

[2] C. Donnet, A. Erdemir, Solid lubricant coatings: Recent developments and future trends, Tribology Letters 17 (3) (2004) 389-397. doi:10.1023/B:TRIL.0000044487.32514.1d.

URL https://link.springer.com/article/10.1023\%2FB\%3ATRIL.0000044487.32514.1d

(C) 2017. This manuscript version is made available under the CC-BY-NC-ND 4.0 license http://creativecommons.org/licenses/by-nc-nd/4.0/ 
[3] G. Levita, A. Cavaleiro, E. Molinari, T. Polcar, M. C. Righi, Sliding properties of MoS 2 layers: Load and interlayer orientation effects, The Journal of Physical Chemistry C 118 (25) (2014) 13809-13816. doi : 10.1021/jp4098099.

URL https://pubs.acs.org/doi/10.1021/jp4098099

[4] Q. Wan, Y. Jin, P. Sun, Y. Ding, Rheological and tribological behaviour of lubricating oils containing platelet $\mathrm{MoS}_{2}$ nanoparticles, Journal of Nanoparticle Research 16 (5) (2014) 2386. doi:10.1007/ s11051-014-2386-2.

URL https://link. springer . com/article/10.1007\%2Fs11051-014-2386-2

[5] B. Podgornik, T. Kosec, A. Kocijan, Č. Donik, Tribological behaviour and lubrication performance of hexagonal boron nitride (h-BN) as a replacement for graphite in aluminium forming, Tribology International 81 (2015) 267-275. doi:10.1016/j.triboint.2014.09.011.

URL http://www.sciencedirect.com/science/article/pii/S0301679X14003351

[6] J. Bares, N. Argibay, P. Dickrell, G. Bourne, D. Burris, J. Ziegert, W. Sawyer, In situ graphite lubrication of metallic sliding electrical contacts, Wear 267 (9) (2009) 1462-1469. doi:10.1016/j.wear. 2009.03 .024$.

URL http://www.sciencedirect.com/science/article/pii/S0043164809002889

[7] The Contact Conundrum, Ch. Topic 7, pp. 193-213. doi:10.1142/9789814656566\_0007. URL https://www.worldscientific.com/doi/abs/10.1142/9789814656566_0007

[8] E. Gnecco, E. Meyer (Eds.), Fundamentals of Friction and Wear on the Nanoscale, NanoScience and Technology, Springer International Publishing, 2015. doi:10.1007/978-3-319-10560-4.

URL https://link. springer.com/book/10.1007\%2F978-3-319-10560-4

[9] Y. Guo, W. Guo, C. Chen, Modifying atomic-scale friction between two graphene sheets: A molecularforce-field study, Phys. Rev. B 76 (2007) 155429. doi:10.1103/PhysRevB.76.155429. URL https://link.aps.org/doi/10.1103/PhysRevB.76.155429

[10] F. Bonelli, N. Manini, E. Cadelano, L. Colombo, Atomistic simulations of the sliding friction of graphene flakes, The European Physical Journal B 70 (4) (2009) 449-459. doi:10.1140/epjb/e2009-00239-7. URL https://link.springer.com/article/10.1140\%2Fepjb\%2Fe2009-00239-7

[11] L. Xu, T.-B. Ma, Y.-Z. Hu, H. Wang, Vanishing stick-slip friction in few-layer graphenes: the thickness effect, Nanotechnology 22 (28) (2011) 285708. doi:10.1088/0957-4484/22/28/285708.

URL https://iopscience.iop.org/article/10.1088/0957-4484/22/28/285708/meta

[12] L. Xu, T. bao Ma, Y. zhong $\mathrm{Hu}, \mathrm{H}$. Wang, Molecular dynamics simulation of the interlayer sliding behavior in few-layer graphene, Carbon 50 (3) (2012) 1025 - 1032. doi:10.1016/j.carbon.2011.10. 006.

URL http://www.sciencedirect.com/science/article/pii/S0008622311008256

(C) 2017. This manuscript version is made available under the CC-BY-NC-ND 4.0 license http://creativecommons.org/licenses/by-nc-nd/4.0/ 
[13] P. Liu, Y. Zhang, A theoretical analysis of frictional and defect characteristics of graphene probed by a capped single-walled carbon nanotube, Carbon 49 (11) (2011) 3687 - 3697. doi:10.1016/j.carbon. 2011.05 .004 .

URL http://www.sciencedirect.com/science/article/pii/S0008622311003484

[14] A. Smolyanitsky, J. P. Killgore, V. K. Tewary, Effect of elastic deformation on frictional properties of few-layer graphene, Phys. Rev. B 85 (2012) 035412. doi:10.1103/PhysRevB.85.035412.

URL https://link.aps.org/doi/10.1103/PhysRevB.85.035412

[15] M. Reguzzoni, A. Fasolino, E. Molinari, M. C. Righi, Friction by shear deformations in multilayer graphene, The Journal of Physical Chemistry C 116 (39) (2012) 21104-21108. doi :10.1021/jp306929g. URL https://pubs.acs.org/doi/10.1021/jp306929g

[16] M. Reguzzoni, A. Fasolino, E. Molinari, M. C. Righi, Potential energy surface for graphene on graphene: Ab initio derivation, analytical description, and microscopic interpretation, Phys. Rev. B 86 (2012) 245434. doi:10.1103/PhysRevB.86.245434.

URL https://link.aps.org/doi/10.1103/PhysRevB.86.245434

[17] O. Hod, Interlayer commensurability and superlubricity in rigid layered materials, Phys. Rev. B 86 (2012) 075444. doi:10.1103/PhysRevB.86.075444.

URL https://link.aps.org/doi/10.1103/PhysRevB.86.075444

[18] I. Leven, D. Krepel, O. Shemesh, O. Hod, Robust superlubricity in graphene/h-BN heterojunctions, The Journal of Physical Chemistry Letters 4 (1) (2013) 115-120. doi:10.1021/jz301758c. URL https://pubs.acs.org/doi/10.1021/jz301758c

[19] S. Cahangirov, S. Ciraci, V. O. Özçelik, Superlubricity through graphene multilayers between Ni(111) surfaces, Phys. Rev. B 87 (2013) 205428. doi:10.1103/PhysRevB.87.205428.

URL https://link.aps.org/doi/10.1103/PhysRevB.87.205428

[20] A. Klemenz, L. Pastewka, S. G. Balakrishna, A. Caron, R. Bennewitz, M. Moseler, Atomic scale mechanisms of friction reduction and wear protection by graphene, Nano Letters 14 (12) (2014) 71457152. doi:10.1021/n15037403.

URL https://pubs.acs.org/doi/10.1021/n15037403

[21] S. Cahangirov, S. Ciraci, Superlubricity in Layered Nanostructures, Springer International Publishing, Cham, 2015, pp. 463-487. doi:10.1007/978-3-319-10560-4_21.

URL https://link.springer.com/chapter/10.1007\%2F978-3-319-10560-4_21

[22] K.-S. Kim, H.-J. Lee, C. Lee, S.-K. Lee, H. Jang, J.-H. Ahn, J.-H. Kim, H.-J. Lee, Chemical vapor deposition-grown graphene: The thinnest solid lubricant, ACS Nano 5 (6) (2011) 5107-5114. doi: 10.1021/nn2011865.

URL https://pubs .acs .org/doi/10.1021/nn2011865

(C) 2017. This manuscript version is made available under the CC-BY-NC-ND 4.0 license http://creativecommons.org/licenses/by-nc-nd/4.0/ 
[23] T. Filleter, J. L. McChesney, A. Bostwick, E. Rotenberg, K. V. Emtsev, T. Seyller, K. Horn, R. Bennewitz, Friction and dissipation in epitaxial graphene films, Phys. Rev. Lett. 102 (2009) 086102. doi:10.1103/PhysRevLett.102.086102.

URL https://link.aps.org/doi/10.1103/PhysRevLett.102.086102

[24] C. Lee, Q. Li, W. Kalb, X.-Z. Liu, H. Berger, R. W. Carpick, J. Hone, Frictional characteristics of atomically thin sheets, Science 328 (5974) (2010) 76-80. doi:10.1126/science.1184167.

URL http://science.sciencemag.org/content/328/5974/76

[25] H. Lee, N. Lee, Y. Seo, J. Eom, S. Lee, Comparison of frictional forces on graphene and graphite, Nanotechnology 20 (32) (2009) 325701. doi:10.1088/0957-4484/20/32/325701.

URL https://iopscience.iop.org/article/10.1088/0957-4484/20/32/325701/meta

[26] Q. Li, C. Lee, R. W. Carpick, J. Hone, Substrate effect on thickness-dependent friction on graphene, physica status solidi (b) 247 (1112) (2010) 2909-2914. doi:10.1002/pssb. 201000555.

URL https://onlinelibrary.wiley.com/doi/abs/10.1002/pssb. 201000555

[27] L.-Y. Lin, D.-E. Kim, W.-K. Kim, S.-C. Jun, Friction and wear characteristics of multi-layer graphene films investigated by atomic force microscopy, Surface and Coatings Technology 205 (20) (2011) 4864 - 4869. doi:10.1016/j.surfcoat.2011.04.092.

URL http://www.sciencedirect.com/science/article/pii/S0257897211004488

[28] D. Marchetto, C. Held, F. Hausen, F. Wählisch, M. Dienwiebel, R. Bennewitz, Friction and wear on single-layer epitaxial graphene in multi-asperity contacts, Tribology Letters 48 (1) (2012) 77-82. doi:10.1007/s11249-012-9945-4.

URL https://link. springer.com/article/10.1007\%2Fs11249-012-9945-4

[29] D. Marchetto, T. Feser, M. Dienwiebel, Microscale study of frictional properties of graphene in ultra high vacuum, Friction 3 (2) (2015) 161-169. doi:10.1007/s40544-015-0080-8.

URL https://link. springer.com/article/10.1007\%2Fs40544-015-0080-8

[30] S. S. Kandanur, M. A. Rafiee, F. Yavari, M. Schrameyer, Z.-Z. Yu, T. A. Blanchet, N. Koratkar, Suppression of wear in graphene polymer composites, Carbon 50 (9) (2012) 3178 - 3183, festschrift dedicated to Peter A. Thrower, Editor-in-Chief, 1972 - 2012. doi:10.1016/j.carbon.2011.10.038. URL http://www.sciencedirect.com/science/article/pii/S0008622311008682

[31] D. Berman, A. Erdemir, A. V. Sumant, Graphene: a new emerging lubricant, Materials Today 17 (1) (2014) 31 - 42. doi:10.1016/j.mattod.2013.12.003.

URL http://www.sciencedirect.com/science/article/pii/S1369702113004574

[32] D. Berman, A. Erdemir, A. V. Sumant, Graphene as a protective coating and superior lubricant for electrical contacts, Applied Physics Letters 105 (23) (2014) 231907. doi:10.1063/1.4903933.

URL https://aip.scitation.org/doi/10.1063/1.4903933

(C) 2017. This manuscript version is made available under the CC-BY-NC-ND 4.0 license http://creativecommons.org/licenses/by-nc-nd/4.0/ 
[33] J. Ota, S. K. Hait, M. I. S. Sastry, S. S. V. Ramakumar, Graphene dispersion in hydrocarbon medium and its application in lubricant technology, RSC Adv. 5 (2015) 53326-53332. doi:10.1039/ C5RA06596H.

URL https://pubs.rsc.org/en/Content/ArticleLanding/2015/RA/C5RA06596H

[34] S. Liang, Z. Shen, M. Yi, L. Liu, X. Zhang, S. Ma, In-situ exfoliated graphene for high-performance water-based lubricants, Carbon 96 (2016) 1181 - 1190. doi:10.1016/j.carbon.2015.10.077. URL http://www.sciencedirect.com/science/article/pii/S0008622315303870

[35] J. Lin, L. Wang, G. Chen, Modification of graphene platelets and their tribological properties as a lubricant additive, Tribology Letters 41 (1) (2011) 209-215. doi:10.1007/s11249-010-9702-5. URL https://link. springer . com/article/10.1007\%2Fs11249-010-9702-5

[36] H. Huang, J. Tu, L. Gan, C. Li, An investigation on tribological properties of graphite nanosheets as oil additive, Wear 261 (2) (2006) 140 - 144. doi:10.1016/j.wear.2005.09.010.

URL http://www.sciencedirect.com/science/article/pii/S0043164805004710

[37] X. Fan, L. Wang, Graphene with outstanding anti-irradiation capacity as multialkylated cyclopentanes additive toward space application, Scientific Reports 5 (2015) 12734. doi:10.1038/srep12734.

URL https://www. nature.com/articles/srep12734

[38] J. Pu, Y. Mo, S. Wan, L. Wang, Fabrication of novel graphenefullerene hybrid lubricating films based on self-assembly for MEMS applications, Chem. Commun. 50 (2014) 469-471. doi:10.1039/C3CC47486K. URL https://pubs.rsc.org/en/Content/ArticleLanding/2014/CC/C3CC47486K

[39] F. Mao, U. Wiklund, A. M. Andersson, U. Jansson, Graphene as a lubricant on ag for electrical contact applications, Journal of Materials Science 50 (19) (2015) 6518-6525. doi:10.1007/ s10853-015-9212-9.

URL https://link. springer.com/article/10.1007\%2Fs10853-015-9212-9

[40] X. Feng, S. Kwon, J. Y. Park, M. Salmeron, Superlubric sliding of graphene nanoflakes on graphene, ACS Nano 7 (2) (2013) 1718-1724. doi:10.1021/nn305722d.

URL https://pubs.acs.org/doi/10.1021/nn305722d

[41] Z. Deng, A. Smolyanitsky, Q. Li, X.-Q. Feng, R. J. Cannara, Adhesion-dependent negative friction coefficient on chemically modified graphite at the nanoscale, Nature Materials 11 (2012) 1032-1037. doi: $10.1038 /$ nmat3452.

URL https://www. nature.com/articles/nmat3452

[42] D. Berman, A. Erdemir, A. V. Sumant, Few layer graphene to reduce wear and friction on sliding steel surfaces, Carbon 54 (2013) 454 - 459. doi:10.1016/j.carbon.2012.11.061.

URL http://www.sciencedirect.com/science/article/pii/S0008622312009529

[43] D. Berman, A. Erdemir, A. V. Sumant, Reduced wear and friction enabled by graphene layers on sliding

(C) 2017. This manuscript version is made available under the CC-BY-NC-ND 4.0 license http://creativecommons.org/licenses/by-nc-nd/4.0/ 
steel surfaces in dry nitrogen, Carbon 59 (2013) 167 - 175. doi:10.1016/j.carbon.2013.03.006.

URL http://www.sciencedirect.com/science/article/pii/S0008622313002108

[44] Y. J. Shin, R. Stromberg, R. Nay, H. Huang, A. T. Wee, H. Yang, C. S. Bhatia, Frictional characteristics of exfoliated and epitaxial graphene, Carbon 49 (12) (2011) 4070 - 4073. doi : 10.1016/j . carbon. 2011. 05.046 .

URL http://www.sciencedirect.com/science/article/pii/S000862231100409X

[45] F. Wählisch, J. Hoth, C. Held, T. Seyller, R. Bennewitz, Friction and atomic-layer-scale wear of graphitic lubricants on $\mathrm{SiC}(0001)$ in dry sliding, Wear 300 (1) (2013) 78 - 81. doi:10.1016/j. wear. 2013.01 .108$.

URL http://www.sciencedirect.com/science/article/pii/S0043164813001300

[46] P. Restuccia, M. Righi, Tribochemistry of graphene on iron and its possible role in lubrication of steel, Carbon 106 (2016) 118 - 124. doi:10.1016/j.carbon.2016.05.025.

URL http://www.sciencedirect.com/science/article/pii/S0008622316303797

[47] A. C. Ferrari, J. C. Meyer, V. Scardaci, C. Casiraghi, M. Lazzeri, F. Mauri, S. Piscanec, D. Jiang, K. S. Novoselov, S. Roth, A. K. Geim, Raman spectrum of graphene and graphene layers, Phys. Rev. Lett. 97 (2006) 187401. doi:10.1103/PhysRevLett.97.187401.

URL https://link.aps.org/doi/10.1103/PhysRevLett.97.187401

[48] P. A. Khomyakov, G. Giovannetti, P. C. Rusu, G. Brocks, J. van den Brink, P. J. Kelly, First-principles study of the interaction and charge transfer between graphene and metals, Phys. Rev. B 79 (2009) 195425. doi:10.1103/PhysRevB.79.195425.

URL https://link.aps.org/doi/10.1103/PhysRevB.79.195425

[49] N. A. Vinogradov, A. A. Zakharov, V. Kocevski, J. Rusz, K. A. Simonov, O. Eriksson, A. Mikkelsen, E. Lundgren, A. S. Vinogradov, N. Mårtensson, A. B. Preobrajenski, Formation and structure of graphene waves on Fe(110), Phys. Rev. Lett. 109 (2012) 026101. doi:10.1103/PhysRevLett.109. 026101.

URL https://link.aps.org/doi/10.1103/PhysRevLett.109.026101

(C) 2017. This manuscript version is made available under the CC-BY-NC-ND 4.0 license http://creativecommons.org/licenses/by-nc-nd/4.0/ 